УДК [616.711.6+616.728.2]-07-089

DOI: $10.26435 /$ UC.V0I3(36).538

\title{
А.Н.Лихолетов
}

ГОО ВПО «Донецкий национальный медицинский университет имени М. Горького», Донецк

\section{СОЧЕТАННОЕ ДЕГЕНЕРАТИВНО-ДИСТРОФИЧЕСКОЕ ПОРАЖЕНИЕ ПОЯСНИЧНО-КРЕСТЦОВОГО ОТДЕЛА ПОЗВОНОЧНИКА И ТАЗОБЕДРЕННЫХ СУСТАВОВ. ТРУДНОСТИ ДИАГНОСТИКИ И ЛЕЧЕНИЯ НIP-SPINЕ СИНДРОМА}

Дегенеративно-дистрофические поражения крупных суставов и позвоночника являются самыми распространенными заболеваниями настоящего времени. В развитых странах мира эта патология занимает второе место после респираторных заболеваний среди причин острой и хронической нетрудоспособности населения $[1,2]$, встречается независимо от возраста индивидов (среди детей, лиц трудоспособного возраста, пожилых людей) [2]. В возрасте старше 45 лет почти 100\% людей ощущают однократную, рецидивирующую или постоянную боль в спине, которая относится к наиболее частым причинам временной нетрудоспособности. По данным американских исследований, больные с такой патологией составляют 17\% всех работников, получающих пособие по нетрудоспособности, 7\% неорганизованной части населения, на них приходится $14 \%$ всех визитов к врачу и $19 \%$ госпитализаций; с целью восстановления функции суставов проводится 10\% всех хирургических операций [1-3]. Любые патологии позвоночника сопровождаются болевым синдромом за исключением случаев нарушения чувствительности. Неприятные ощущения в области спины отличаются многокомпонентностью и широким спектром возможных причин [3] и соответственно высокой распространенностью (встречается у 60-80\% людей) $[1,4]$. Приобретенные патологии позвоночника и суставов часто возникают под действием неблагоприятных статико-динамических условий, приводящих к компенсаторной перестройке позвоночно-двигательного сегмента. Поэтому в странах на постсоветском пространстве врачи часто пользуются собирательным термином «остеохондроз позвоночника», который в медицине стран западной Европы и США не имеет аналогичных терминов [5]. Данное понятие объединяет большое количество различных патологических состояний. В результате постановка диагноза по международной классифи- кации болезней порождает массу неточностей и ошибок [6]. Дегенеративно-дистрофические заболевания позвоночника и тазобедренных суставов зачастую имеют схожую клиникорентгенологическую картину, сопровождаются болевым синдромом с двигательными нарушениями и многими авторами рассматриваются как единый патогенетический процесс [7]. Значительная частота поражений поясничнокрестцового отдела позвоночника и пояса нижних конечностей связана с рядом особенностей, среди которых большое значение имеют конституциональные факторы, в частности строение позвонков, связочного аппарата и позвоночника в целом, возрастные изменения межпозвонковых дисков, особенности испытываемых нагрузок и биомеханики позвоночника, таза, суставов нижних конечностей.

Поэтому разработка концептуальных моделей диагностики различной патологии позвоночника и пояса нижних конечностей обусловлена большим количеством пациентов, ростом заболеваемости, отсутствием единого мнения специалистов к лечебно-диагностическим методикам, требующим мультидициплинарного подхода.

Разработка концептуальных моделей обследования и лечения пациентов с болью в спине может улучшить эффективность коррекции и устранения выявленной патологии. Сочетанная патология позвоночника и суставов - проблема, безусловно требующая участия разных специалистов. Болевой синдром, в данном случае, обладает выраженной неспецифичностью. Кроме алгоритмов диагностики вертеброгенной патологии и патологии суставов необходимо исключить у пациента поражения соединительной ткани, заболевания внутренних органов, включая такие патологии как синдром конского хво-

(c) А.Н. Лихолетов, 2020

(c) Университетская Клиника, 2020 
ста, поражения в результате развития опухоли, воспаления, инфекции, травмы, системный остеопороз. Необходимо установить, что является причиной синдрома: вышеуказанные заболевания, компрессионная радикулопатия или скелетно-мышечная боль в спине [8]. Дифференциальный диагноз боли в спине в амбулаторных условиях включает широкий спектр причин, в частности спондилез, перелом тела позвонка, мышечные спазмы. Наличие фебрильного статуса у пациента не доказывает развитие инфекционного заболевания позвоночника (остеомиелита, в частности). Для объективизации оценки локомоторных нарушений в клинической практике широко используют биомеханические исследования, двигательные тесты и тестирующие нагрузки. Однако наиболее информативным и физиологичным диагностическим тестом функционального состояния опорно-двигательной системы до сих пор остается ходьба $[1,7]$. Степень нарушения ходьбы зависит не только от выраженности патологических изменений в более страдающем тазобедренном суставе, но и от состояния контрлатеральной конечности. Изменения биомеханических параметров ходьбы по мере прогрессирования коксартроза соответствуют динамике клинической оценки, при этом величина нагрузки на больную ногу снижается и становится неравномерной [9].

Вместе с тем анализ литературных данных свидетельствует о том, что функциональное состояние локомоторной системы при заболеваниях поясничного отдела позвоночника и тазобедренных суставов изучается раздельно друг от друга. При этом отмечено, что частота встречаемости сочетанной патологии при дегенеративно-дистрофических заболеваниях позвоночника и тазобедренных суставов превышает 28,5\%, особенно среди лиц пожилого возраста. В свою очередь сочетанное поражение тазобедренных суставов и пояснично-крестцового отдела позвоночника приводит к взаимному отягощению и рассматривается как hip-spine синдром (HSS) [7]. Под данным термином понимается полиэтиологичный симптомокомплекс, который характеризуется болевым синдромом, функциональными нарушениями и изменением анатомо-биомеханических взаимоотношений в системе «тазобедренный сустав - позвоночник» вследствие развития миодистрофических, нейрогенных синдромов, что приводит к возникновению или прогрессированию дистрофических изменений [10]. Термин «hip-spine syndrome» был введен C. Offierski и I. Macnab в 1983 году, которые создали классификацию и выделили три типа HSS - простой, сложный и вторичный $[11,12]$. Простой тип характеризует- ся патологическими изменениями и в поясничном отделе, и в тазобедренном суставе, но клинически доминирует один источник боли. У пациентов со сложным типом HSS не удается выделить однозначный источник боли. При вторичном HSS оба патологических процесса усугубляют течение друг друга. Болевой синдром, обусловленный дегенеративным стенозом позвоночного канала, может имитировать патологию тазобедренного сустава, имея сходную локализацию $[11,13,14]$. Степень распространенности симптоматического стеноза позвоночного канала в поясничном отделе увеличивается с возрастом и обычно затрагивает лиц старше 60 лет $[11,12,15]$. Среди всех нейрохирургических операций у лиц старше 65 лет большинство выполняется именно по поводу дегенеративного стеноза позвоночного канала. По данным литературы от 68 до 98\% лиц преклонного возраста имеют те, или иные морфологические изменения позвоночного столба [10, 16, 17].

Патология поясничного отдела позвоночника и остеоартроз тазобедренного сустава могут проявляться болевым синдромом в этой же области, что приводит к дополнительным сложностям в диагностике [18]. Рентгенологические методы диагностики обязательно используются при неясном источнике боли. Они позволяют выявить изменения в тазобедренном суставе и поясничном отделе позвоночника, что ведет к более полному пониманию этиологии жалоб у пациентов $[11,18,19]$. В рамках HSS клинический интерес представляет обнаружение двух патологий - остеоартроза тазобедренного сустава и стеноза пояснично-крестцового отдела позвоночника. Причина жалоб при наличии обеих патологий может быть не очевидна и привести к ошибкам в выборе тактики лечения [20, 21]. В медицине часто оперируют понятием как «хроническая тазовая боль» (ХТБ) [22]. Часто данный синдром является причиной патологий гинекологического или урологического характера [23], но широко распространено явление неустановленной ХТБ [24]. Постановка диагноза отличается сложностью в связи с тесными взаимосвязями (посредством иннервации, кровообращения, мышц и связок) органов малого таза. Поэтому патологический процесс распространяется и на мышечно-суставной аппарат тазового пояса [25]. ХТБ является примером централизованных болевых синдромов, в которых соматическая и висцеральная система могут участвовать в восприятии боли, что затрудняет дифференциальную диагностику. В практической медицине и научной литературе мало внимания уделяется патологии тазобедренных суставов региона, что может привести к длительной диагностике па- 
циента с существующей проблеме в данной области [6]. При этом доля пациентов с БНЧС с патологиями в крестцово-подвздошных сочленениях (КПС) составляет от 10-25\% [26]. Хронизация процесса может привести к псевдокорешковым болям и ошибочным нейрохирургическим операциям по устранению компрессии позвоночника (ламинэктомиям). Под проявлениями болевого синдрома в пояснично-крестцовом отделе позвоночника часто скрываются тазовые патологии, поэтому такой термин как «люмбосакралгия» в будущем может стать официальным рабочим диагнозом [6, 25, 27].

В связи с широким разнообразием этиологии болевых синдромов дискогенной, артрогенной и/или миофасциальной природы актуальным вопросом является дифференциальная диагностика причин их проявления в КПС [28]. Отсутствие высокочувствительных и специфических методов диагностики патологии КПС приводит к необходимости применения совокупности провокационных клинических тестов. При получении 3 или более положительных результатов чувствительность и специфичность диагностики составляет до 85\% [29]..Среди всех вариантов диагностики выделяют тест Генслена с чувствительностью и валидностью до $81 \%$. Его предлагают использовать в комплексе с подтверждением диагноза посредством диагностической блокады под контролем рентгенографии даже при наличии отрицательных результатов других тестов. Максимальная чувствительность (97\%) достигается при использовании таких групп тестов как упругость бедра Патрика и Ганслена. Данные комбинации обеспечивают получение высокой достоверности наличия/отсутствия синдрома крестцово-подвздошных сочленений и дифференцирование его с диско- и артрогенной патологиями поясничного отдела позвоночника или дегенеративными заболеваниями тазобедренных суставов [30-32].

Рентгенологические исследования широко используются при патологии тазобедренного сустава и поясничного отдела позвоночника, тем не менее, связь рентгенологических изменений и клинической картины не высока $[33,34]$. В отечественной литературе есть данные об отсутствии взаимосвязи рентгенологических критериев и клиники стеноза поясничного отдела позвоночника [35]. Если пациентам неправильно диагностирован источник боли и проведена операция, последующее вмешательство может переноситься более тяжело [36]. Проблема хирургического лечения дегенеративнодистрофических заболеваний поясничного отдела позвоночника при HSS продолжает оставаться весьма актуальной [37], что подтвержда- ется появлением множества печатных работ как в отечественной, так и в зарубежной печати $[1,2$, 38-40]. С появлением новой генераций имплантатов и материалов для эффективных методик анестезиологического обеспечения, существенно расширились показания к оперативному лечению у данной категории больных. При лечении данной категории пациентов, большинство авторов отмечают преобладание многоуровневых поражений, которые требуют проведения декомпрессивных вмешательств зачастую с необходимостью стабилизации и реконструкции. $[12,41]$. При любом варианте развития HSS возникает вопрос о проведении оперативного вмешательства на позвоночнике или тазобедренном суставе. Даже после успешно выполненной операции нередко прогрессируют симптомы поражения позвоночного сегмента или суставов. Это проявляется болевым синдромом и часто нивелирует результаты артропластики, так как восстановление движений в тазобедренном суставе, изменение длины и опороспособности конечности после операции тотального эндопротезирования ведёт к разрыву сформировавшегося функционального стереотипа, изменению подвижности поясничного отдела позвоночника, регрессу перекоса таза, что вызывает динамическую компрессию корешков спинномозговых нервов [42]. Если опираться на клиническую картину и на рентгенологические изменения, то операция на тазобедренном суставе приводит к улучшению состояния не более чем в $75 \%$ случаев $[1,13]$. Результаты исследования 1000 пациентов в разные сроки после тотального эндопротезирования тазобедренного сустава доказали, что боли в пояснично-крестцовом отделе позвоночника сохраняются у $15,1 \%$ из них, а у $14,9 \%$ возникают новые болевые ощущения даже после успешно выполненной операции тотального эндопротезирования тазобедренного сустава. Более того, нередко прогрессируют и симптомы поражения люмбо-сакральных позвоночнодвигательных сегментов, проявляющиеся болевым синдромом и часто нивелирующие результаты артропластики [43]. Среди факторов, значительно снижающих качество жизни больных после эндопротезирования тазобедренного сустава, болевому синдрому в поясничном отделе позвоночника принадлежит одна из главенствующих ролей $[7,44]$.

При сочетанном поражении позвоночника и тазобедренного сустава последовательность оперативного лечения определяется на основании превалирования патологии в том или ином сегменте. [45]. Лучшие функциональные результаты лечения коксартроза методом эндопротезирования достигаются до развития значимых 
нарушений анатомии тазобедренного сустава, перекоса таза, сколиоза и проявления неврологической симптоматики [46]. Исследования 141 пациента после эндопротезирования тазобедренного сустава по поводу диспластического коксартроза показало, что исход оперативного лечения нередко зависит от биомеханических параметров позвоночника. При ограничении объема движений сгибание-разгибание позвоночника прогноз эндопротезирования не всегда является благоприятным [47].

Несмотря на явные успехи в хирургическом лечении дегенеративных заболеваний поясничного отдела позвоночника у возрастных пациентов, остается ряд нерешенных вопросов [48]. На настоящий момент не уточнен вид и объем оперативных вмешательств при каждой конкретной нозологии и при их сочетании. С одной стороны предлагаются миниинвазивные вмешательства с максимально возможным сохранением опорных структур [39, 41.], другие авторы пропагандируют широкие декомпрессивные вмешательства [12, 49]. Активно дискутируется вопросы о необходимости и методе стабилизации позвоночно-двигательного сегмента при каждом виде нозологий, особенно в условиях остеопороза. Фактически каждый клинический случай сочетает в себе различные нозологические дефиниции, в особенности при многоуровневом поражении $[40,48]$, что требует избирательного подхода к выбору хирургического вмешательства у данной категории больных. При этом целью хирургического лечения должна быть максимально возможная декомпрес- сия и реконструкция позвоночно-двигательного сегмента с учетом стадии и распространенности дегенеративных изменений.

\section{З А К Л Ю Ч Н ИЕ}

Таким образом, несмотря на прогресс в изучении отдельных дегенеративно-дистрофических поражений опорно-двигательной системы, отсутствует однозначное решение проблемы hipspine синдрома. Чаще упоминается о поражении тазобедренного сустава как о «первичном» компоненте, чем о вертеброгенном. Остаются открытыми вопросы дифференциальной диагностики и определения взаимосвязи биомеханических нарушений дегенеративнодистрофических поражений поясничнокрестцового отдела позвоночника и тазобедренных суставов. До сих пор не существует единого мнения в выборе методики оперативного вмешательства при сочетанной патологии тазобедренного сустава и пояснично-крестцового отдела позвоночника, при котором нейрохирурги и травматологи-ортопеды зачастую упускают из виду смежные отделы, патология которых может маскировать симптомы основного заболевания. Таким образом, при болях, связанных с поражением пояснично-крестцового отдела позвоночника и пояса нижних конечностей необходимо дифференцировать вертеброгенные или суставные боли от синдромов другой этиологии (при поражении мягких тканей, внутренних органов). Только при однозначном определении источника боли можно рассчитывать на успех хирургического лечения.

\section{А.Н. Лихолетов}

ГОО ВПО «Донецкий национальный медицинский университет имени М. Горького», Донецк

\section{СОЧЕТАННОЕ ДЕГЕНЕРАТИВНО-ДИСТРОФИЧЕСКОЕ ПОРАЖЕНИЕ ПОЯСНИЧНО-КРЕСТЦОВОГО ОТДЕЛА ПОЗВОНОЧНИКА И ТАЗОБЕДРЕННЫХ СУСТАВОВ. ТРУДНОСТИ ДИАГНОСТИКИ И ЛЕЧЕНИЯ НIP-SPINЕ СИНДРОМА}

Дегенеративно-дистрофические поражения крупных суставов и позвоночника являются самыми распространенными заболеваниями настоящего времени, зачастую имеют схожую клинико-рентгенологическую картину, сопровождаются болевым синдромом с двигательными нарушениями и многими авторами рассматривается как hip-spine синдром. В статье приведен обзор литературы о сложностях дифференциальной диагностики и лечения сочетанного дегенеративно-дистрофического поражения тазобедренных суставов и пояснично-крестцового отдела позвоночника. В литературе чаще упоминается о поражении тазобедренного сустава как о «первичном» компоненте, чем о вертеброгенном. Разработка концептуальных моделей диагностики обусловле- ны большим количеством пациентов, ростом заболеваемости, отсутствием единого мнения специалистов к лечебно-диагностическим методикам, требующим мультидициплинарного подхода. До сих пор не уточнены вид и объем оперативных вмешательств при каждой конкретной нозологии и при их сочетании. Необходимы в данном вопросе разработка таких диагностических алгоритмов, при которых возможно однозначное определение источника боли и позволит успешно реализовать хирургическое лечение с восстановлением кинематических взаимоотношений в системе «позвоночник-таз-нижние конечности».

Ключевые слова: дегенеративно-дистрофические поражения крупных суставов и позвоночника, hipspine синдром, эндопротезирование. 


\section{A.N. Likholetov}

\section{SEI HPE «M. Gorky Donetsk National Medical University», Donetsk}

\section{COMBINED DEGENERATIVE-DYSTROPHIC DEFRACTION OF THE LUMBASACRAL SPINE AND HIP JOINTS. DIFFICULTIES OF DIAGNOSIS AND TREATMENT OF HIP-SPINE SYNDROME}

Degenerative-dystrophic lesions of large joints and spine are the most common diseases of the present, often have a similar clinical and radiological picture, are accompanied by pain syndrome with motor disorders, and many authors consider it as hip-spine syndrome. The article provides a review of the literature on the difficulties of differential diagnosis and treatment of combined degenerative-dystrophic lesions of the hip joints and lumbosacral spine. In the literature, it is more often mentioned that the hip joint is damaged as a "primary" component than vertebrogenic. The development of conceptual diagnostic models is due to a large number of patients, an increase in the incidence rate, and the lack of a unified opinion of specialists on diagnostic and treatment methods that require a multidisciplinary approach. The type and volume of surgical interventions for each specific nosology and their combination have not yet been clarified. The development of such diagnostic algorithms is necessary in this matter, in which an unambiguous determination of the source of pain is possible and will allow the successful implementation of surgical treatment with the restoration of kinematic relationships in the "spine-pelvis-lower limbs" system.

Key words: degenerative-dystrophic lesions of large joints and spine, hip-spine syndrome, arthroplasty.

\section{ЛИТЕРАТУРА}

1. Хвисюк А.Н. Тазобедренно-поясничный синдром (патогенез, диагностика, принципы лечения) : дис....д-ра мед. наук. Харьков; 2002; 114-119.

2. Raciborski F, Gasik R, Kłak A. Disorders of the spine. A major health and social problem. Reumatologia. 2016; 54 (4): 196-200. DOI: 10.5114/reum.2016.62474

3. Eloqayli H. Clinical Decision-Making in Chronic Spine Pain: Dilemma of Image-Based Diagnosis of Degenerative Spine and Generation Mechanisms for Nociceptive, Radicular, and Referred Pain. Biomed Res Int. 2018; 43: 1-6. DOI:10.1155/2018/8793843

4. Lee C.H., Chung C.K., Kim C.H., Kwon J.W. Health Care Burden of Spinal Diseases in the Republic of Korea: Analysis of a Nationwide Database From 2012 through 2016. Neurospine. $2018 ; 15$ (1): 66-76. DOI:10.14245/ns.1836038.019

5. Петров К.Б. Остеохондроз позвоночника: в защиту нозологической, терминологической и классификационной индивидуальности. Медицина в Кузбассе. 2012; 1: 52-58.

6. Ахметов Б.Х., Максимов Ю.Н., Хайбуллина Д.Х., Губеев Б.Э. Боли в нижней части спины: нюансы диагностики. Практическая медицина. 2014; 2 (78): 17-20.

7. Негреева М.Б., Ларионов С.Н., Сороковиков В.А., Шендеров В.А. Биомеханические аспекты исследований дегенеративно-дистрофичкских заболеваний поясничного отдела позвоночника и тазобедренных суставов (обзор литературы). Бюллетень ВСЦН СО РАМН. 2013; 5 (93): 187-190.

8. Садоха К.А., Головко А.М., Кротов В.В. Боль в спине: причины возникновения, диагностика, лечение, современный взгляд на проблему. Медицинские новости. 2018; 1 (280): 63-68.

9. Михайлюк, И. Г. Анализ корректности диагностики причин боли в области спины в неврологическом стационаре в соответствии с международной классификацией болезней. Нервно-мышечные болезни. 2014; 3 : 20-27.

10. Шурова Е.Н., Хомченков М.В., Камшилов Б.В, и др. Дифференциальная диагностика hip-spine синдрома на этапе предоперационной подготовки. Гений ортопедии. 2012; 4: 125-127.

11. Месхи К.Т., Каргальцев А.А., Макаров М.А., Ворона Б.Н. Лучевые методы визуализации при коксовертебральном синдроме в рамках дифференциальной диагностики источника боли. REJR 2018; 8 (4): 220-228. DOI: $10.21569 / 2222-7415-2018-8-4-220-228$

12. Offierski CM, MacNab I. Hip-spine syndrome. Spine.

\section{REFERENCES}

1. Hvisjuk A.N. Tazobedrenno-pojasnichnyj sindrom (patogenez, diagnostika, principy lechenija) : dis....d-ra med. nauk. Har'kov; 2002: 114-119 (in Russian).

2. Raciborski F, Gasik R, Kłak A. Disorders of the spine. A major health and social problem. Reumatologia. 2016; 54 (4): 196-200. DOI:10.5114/reum.2016.62474

3. Eloqayli H. Clinical decision-making in chronic spine pain: dilemma of image-based diagnosis of degenerative spine and generation mechanisms for nociceptive, radicular, and referred pain. // Biomed Res Int. 2018; 43: 1-6. DOI:10.1155/2018/8793843

4. Lee C.H., Chung C.K., Kim C.H., Kwon J.W. Health Care Burden of Spinal diseases in the republic of Korea: Analysis of a Nation wide database from 2012 Through 2016. Neurospine. 2018; 15 (1): 66-76. DOI:10.14245/ns.1836038.019

5. Petrov K.B. Osteohondroz pozvonochnika: v zashhitu nozologicheskoj, terminologicheskoj i klassifikacionnoj individual'nosti. Medicina v Kuzbasse. 2012; 1: 52-58 (in Russian).

6. Ahmetov B.H., Maksimov Ju.N., Hajbullina D.H., Gubeev B.Je. Boli v nizhnej chasti spiny: njuansy diagnostiki. Prakticheskaja medicina. 2014; 2 (78): 17-20 (in Russian).

7. Negreeva M.B., Larionov S.N., Sorokovikov V.A., Shenderov V.A. Biomehanicheskie aspekty issledovanij degenerativno-distrofichkskih zabolevanij pojasnichnogo otdela pozvonochnika i tazobedrennyh sustavov (obzor literatury)ю Bjulleten’ VSCN SO RAMN. 2013; 5 (93): 187-190 (in Russian).

8. Sadoha K.A., Golovko A.M., Krotov V.V. Bol' v spine: prichiny vozniknovenija, diagnostika, lechenie, sovremennyj vzgljad na problemu. Medicinskie novosti. 2018; 1 (280): 63-68 (in Russian).

9. Mihajljuk, I. G. Analiz korrektnosti diagnostiki prichin boli v oblasti spiny v nevrologicheskom stacionare v sootvetstvii s mezhdunarodnoj klassifikaciej boleznej. Nervno-myshechnye bolezni. 2014; 3: 20-27 (in Russian).

10. Shhurova E.N., Homchenkov M.V., Kamshilov B.V, i dr. Differencial'naja diagnostika hip-spine sindroma na jetape predoperacionnoj podgotovki. Genij ortopedii. 2012; 4: 125-127 (in Russian).

11. Meshi K.T., Kargal'cev A.A., Makarov M.A., Vorona B.N. Luchevye metody vizualizacii pri koksovertebral'nom sindrome v ramkah differencial'noj diagnostiki istochnika boli. REJR 2018; 8 (4): 220-228. DOI:10.21569/2222-7415 -2018-8-4-220-228 (in Russian).

12. Offierski CM, MacNab I. Hip-spine syndrome. Spine. 1983; 8 (3): 316. 
1983; 8 (3): 316

13. Jonathan N. Sembrano, David W. Polly, Jr, How Often Is Low Back Pain Not Coming From the Back, SPINE. 2008; 34 (1): 27-32.

14. Massimo Allegri et al., Mechanisms of low back pain: a guide for diagnosis and therapy. 2016; 285 (F1000 Faculty Rev): 1530 .

15. Deyo R.A, Mirza S.K., Martin B.I., Kreuter W., Goodman D.C., Jarvik J.G. Trends, major medical complications, and charges associated with surgery for lumbar spinal stenosis in older adults. JAMA. 2010; 303 (13): 1259-1265.

16. Макиров С.К. Хирургическое лечение структурнофункциональных нарушений при остеохондрозе позвоночника. Диссертация на соискание ученой степени д.м.н. М.; 2006: 72-79.

17. Schwab, Frank; Benchick el-Fegoun, Abdelkrim; Gamez, Lorenzo; Goodman, Howard; Farcy, Jean-Pierre A lumbar classification of Scoliosis in the Adult Patient: Preliminary Approach. Spine. 2005; Vol. 30, 14: 1670-1673.

18. Devin C.J. et al. Hip-spine syndrome. J Am Acad Orthop Surg. 2012; 20 (7): 434-442.

19. Redmond J.M., Gupta A., Nasser R., Domb B.G. The hipspine connection: understanding its importance in the treatment of hip pathology. Orthopedics. 2015; 38 (1): 49.

20. Brown M.D., Gomez-Martin O., Brookfield K.F., et al. Differential diagnosis of hip disease versus spine disease. Clin Orthop 2004; 1: 280.

21. Khan A.M., McLoughlin E. et al. Hip osteoarthritis: where is the pain? Ann R Coll Surg Engl. 2004; 86 (2): 119-21.

22. Covaro A, Vilà-Canet G, de Frutos AG, Ubierna MT, Ciccolo F, Caceres E. Management of degenerative lumbar spinal stenosis: an evidence-based review. EFORT Open Rev. 2017; 1 (7): 267-274. Published 2017 Mar 13. DOI:10.1302/2058-5241.1.000030

23. Lee BI, Cha HG, Lee WH. The effects of sitting with the right leg crossed on the trunk length and pelvic torsion of healthy individuals. J PhysTherSci. 2016; 28 (11): 31623164. DOI:10.1589/jpts.28.3162

24. Asiri, MD, Banjar R., Al-Qahtani, W. et al. Изменения центральной нервной системы у пациентов с воспалением таза / болью. Curr Bladder Dysfunct Rep 14, 223-230 (2019). D OI: 10.1007/s11884-019-00530-х

25. Баринов А.Н. Диагностика и лечение тазовой боли. Медицинский Совет. 2015; (10): 66-76. DOI: 10.21518/2079-701X-2015-10-66-76

26. Raj MA, Varacallo M. Sacroiliac (SI) Joint Pain. 2019 May 12. StatPearls [Internet]. Treasure Island (FL): StatPearls Publishing; 2020. URL: http://www.ncbi.nlm.nih.gov/ books/NBK470299/PubMed PMID: 29261980

27. Баринов А.Н., Рожков Д.О. Диагностика и лечение ноципластической скелетно-мышечной боли. Медицинский алфавит. 2018; 3 (27): 5-12.

28. Manchikanti L, Abdi S, Atluri S, et. An update of comprehensive evidence-based guidelines for interventional techniques in chronic spinal pain. Part II: Guidance and recommendations. Pain Physician. 2013; 16 (2 Suppl): S49-283.

29. Дамулин И.В. Боль в нижней части спины: клинические особенности и обследование больных. Неврология, нейропсихиатрия, психосоматика. 2014; 1: 9-15.

30. Passavanti MB, Pota V, Sansone P, Aurilio C, De Nardis L, Pace MC. Chronic Pelvic Pain: Assessment, Evaluation, and Objectivation. Pain Res Treat. 2017: 925-947.

31. Casser HR, Seddigh S, Rauschmann M. Acute Lumbar Back Pain. Dtsch Arztebl Int. 2016; 113 (13): 223-234. DOI: 10.3238/arztebl.2016.0223

32. Смоланка В.И., Федурця В.М., Павлов Б.Б. Эпидуральное введение кортикостероидов в лечении хронических дискогенных болей в нижней части спины. Боль. Суставы. Позвоночник. 2019; 1: 59-64.

33. Iidaka T1, Muraki S et al. Prevalence of radiographic hip osteoarthritis and its association with hip pain in Japanese men and women: the ROAD study. Osteoarthritis Cartilage. 2016; 24 (1): 117-23.

34. Kinds M.B., Welsing P.M. et al. A systematic review of the association between radiographic and clinical osteoarthri-
13. Jonathan N. Sembrano, David W. Polly, Jr, How Often Is Low Back Pain Not Coming From the Back, SPINE. 2008; 34 (1): 27-32.

14. Massimo Allegri et al., Mechanisms of low back pain: a guide for diagnosis and therapy. 2016; 285 (F1000 Faculty Rev): 1530 .

15. Deyo R.A, Mirza S.K., Martin B.I., Kreuter W., Goodman D.C., Jarvik J.G. Trends, major medical complications, and charges associated with surgery for lumbar spinal stenosis in older adults. JAMA. 2010; 303 (13): 1259-1265.

16. Makirov S.K. hirurgicheskoe lechenie strukturnofunkcional'nyh narushenij pri osteohondroze pozvonochnika. Dissertacija na soiskanie uchenoj stepeni d.m.n. M.; 2006: 72-79 (in Russian).

17. Schwab, Frank; Benchick el-Fegoun, Abdelkrim; Gamez, Lorenzo; Goodman, Howard; Farcy, Jean-Pierre A lumbar classification of Scoliosis in the Adult Patient: Preliminary Approach. Spine. 2005; Vol. 30, 1:1670-1673.

18. Devin C.J. et al. Hip-spine syndrome. J Am Acad Orthop Surg. 2012; 20 (7): 434-442.

19. Redmond J.M., Gupta A., Nasser R., Domb B.G. The hipspine connection: understanding its importance in the treatment of hip pathology. Orthopedics. 2015; 38 (1): 49.

20. Brown M.D., Gomez-Martin O., Brookfield K.F., et al. Differential diagnosis of hip disease versus spine disease. Clin Orthop. 2004; 1: 280

21. Khan A.M., McLoughlin E. et al. Hip osteoarthritis: where is the pain? Ann R Coll Surg Engl. 2004; 86 (2): 119-21.

22. Covaro A, Vilà-Canet G, de Frutos AG, Ubierna MT, Ciccolo F, Caceres E. Management of degenerative lumbar spinal stenosis: an evidence-based review. EFORT Open Rev. 2017; 1 (7): 267-274. DOI:10.1302/2058-5241.1.000030

23. Lee BI, Cha HG, Lee WH. The effects of sitting with the right leg crossed on the trunk length and pelvic torsion of healthy individuals. J PhysTherSci. 2016; 28 (11): $3162-$ 3164. DOI:10.1589/jpts.28.3162

24. Asiri, MD, Banjar R., Al-Qahtani, W. et al. Изменения центральной нервной системы у пациентов с воспалением таза / болью. Curr Bladder Dysfunct. Rep 14; $223-230$ (2019). DOI: 10.1007/s11884-019-00530-x

25. Barinov A.N. Diagnostika i lechenie tazovoj boli. Medicinskij Sovet. 2015; (10): 66-76. DOI: 10.21518/2079-701X2015-10-66-76 (in Russian).

26. Raj MA, Varacallo M. Sacroiliac (SI) Joint Pain. 2019 May 12. StatPearls [Internet]. Treasure Island (FL): StatPearls Publishing; 2020 Jan. Availablefrom http://www.ncbi.nlm. nih.gov/books/NBK470299/PubMed PMID: 29261980.

27. Barinov A.N., Rozhkov D.O. Diagnostika i lechenie nociplasticheskoj skeletno-myshechnoj boli. Medicinskij alfavit. 2018; 3 (27): 5-12 (in Russian).

28. Manchikanti L, Abdi S, Atluri S, et. An update of comprehensive evidence-based guidelines for interventional techniques in chronic spinal pain. Part II: Guidance and recommendations. Pain Physician. 2013; 16 (2 Suppl): 49283.

29. Damulin I,V. Bol' v nizhnej chasti spiny: klinicheskie osobennosti i obsledovanie bol'nyh. Nevrologija, nejropsihiatrija, psihosomatika. 2014; 1: 9-15 (in Russian).

30. Passavanti MB, Pota V, Sansone P, Aurilio C, De Nardis L, Pace MC. Chronic pelvic pain: Assessment, Evaluation, and Objectivation. Pain Res Treat. 2017: 925-947.

31. Casser HR, Seddigh S, Rauschmann M. Acute lumbar back pain. Dtsch Arztebl Int. 2016; 113 (13): 223-234. DOI: 10.3238/arztebl.2016.0223

32. Smolanka V.I., Fedurcja V.M., Pavlov B.B. Jepidural'noe vvedenie kortikosteroidov v lechenii hronicheskih diskogennyh bolej v nizhnej chasti spiny. Bol'. Sustavy. Pozvonochnik. 2019; 1: 59-64 (in Russian).

33. Iidaka T1, Muraki S et al. Prevalence of radiographic hip osteoarthritis and its association with hip pain in Japanese men and women: the ROAD study. Osteoarthritis Cartilage. 2016; 24 (1): 117-23.

34. Kinds M.B., Welsing P.M. et al. A systematic review of the association between radiographic and clinical osteoarthritis of hip and knee. Osteoarthritis Cartilage. 2011; 19 (7): 768-78. 
tis of hip and knee. Osteoarthritis Cartilage. 2011; 19 (7): 768-78.

35. Шевелев И. Н, Корниенко В. Н. и др. Анализ корреляции рентгенологических критериев и клинических проявлений центрального стеноза поясничнокрестцового отдела позвоночного канала. «Вопросы нейрохирургии» им. Н.Н. Бурденко. 2012; 76 (3): 61-68.

36. T. Eneqvist et al., Lumbar surgery prior to total hip arthroplasty is associated with worse patientreported outcomes. Bone Joint J. 2017; 99-B: 759-65.

37. Вакуленко В. М., Климовицкий В. Г., Бублик Л. А. особенности диагностики и лечения коксартроза на фоне дистрофических изменений в пояснично-крестцовом отделе позвоночника. Оригінальні дослідження. 2008; Т. 9. 2: 15-19.

38. Слиняков Л.Ю., Кавалерский Г.М., Макиров С.К., Ченский А.Д., Бобров Д.С. особенности использования интраоперационного рентгенологического контроля при малоинвазивных стабилизирующих операциях на грудном и поясничном отделах позвоночника. Вестник Российской Военно-медицинской академии СПб.: ВМедА. 2009; 4 (28): 116-117.

39. Bresnahan, Lacey; Ogden, Alfred T.; Natarajan, Raghu N.; Fessler, Richard G. A biomechanical Evaluation of graded Posterior Element Removal for treatment of lumbar Stenosis: comparison of a Minimally Invasive Approach With two Standard laminectomy techniques. Spine. 2009; Vol.34., 1: 17-23.

40. Martin, C Ryan; Gruszczynski, Adam T.; Braunsfurth, Heike A.; Fallatah, Salah M.; O'Neil, Joseph; Wai, Eugene K. The Surgical Management of Degenerative lumbar Spondylolisthesis: A Systematic Review Spine. 2007; Vol.32, 16: 1791-1798.

41. Fu, Yi-Shan; Zeng, Bing-Fang; Xu, Jian-Guang long-term outcomes of two Different Decompressive techniques for lumbar Spinal Stenosis. Spine.2008; Vol.33, 5: 514-518.

42. Денисов А.О., Шильников В.А., Барнс С.А. Коксовертебральный синдром и его значение при эндопротезировании тазобедренного сустава: (обзор лит.). Травматология и ортопедия России. 2012; 1: 121-127.

43. Денисов А.О. Болевой синдром после эндопротезирования тазобедренного сустава: автореф. ... канд. мед. наук. СПб; 2010: 25.

44. Ахтямов И.Ф., Гурылева М.Э., Юосеф А.И. и др. Оценка возможных параллелей между показателями качества жизни и клинико-функциональным состоянием у больных после эндопротезирования тазобедренного сустава. Вестник травматологии и ортопедии им. Н.Н. Приорова. 2008; 1:75-79.

45. Вакуленко В.M. Дифференцированный подход к коксалгии на фоне дистрофических изменений в пояснично-крестцовом отделе позвоночника. Международный неврологический журнал. 2008; 2 (18): 152156.

46. Рагозин А.О. Клинико-биомеханические аспекты оптимизации функции тазобедренного сустава при эндопротезировании: автороф. дис. ... канд. мед. наук. М.; 2004: 17.

47. Кацитадзе Т.А. Значимость биомеханических показателей позвоночника для прогноза исхода лечения диспластического коксартроза. Georg. Med. News. 2004; 7-8: 13-15.

48. Кавалерский Г.М., Черепанов В.Г., Коркунов А.Л., Лычагин А.В., Середа А.П.Дегенеративно-дистрофические поражения пояснично-крестцового отдела позвоночника при hip-spine синдроме: хирургическое лечение. Кафедра травматологии и отропедии. 2013; 3 (7):.4-9.

49. Li, Gordon; Patil, Chirag G.; Lad, Shivanand P.; Ho, Chris; Tian, Wendy; Boakye, Maxwell. Effects of age and comorbidities on complication rates and adverse outcomes after lumbar laminectomy in elderly patients. Spine. 2008; Vol. 33, 11: 1250-1255.
35. Shevelev I. N, Kornienko V. N. i dr. Analiz korreljacii rentgenologicheskih kriteriev i klinicheskih projavlenij central'nogo stenoza pojasnichno-krestcovogo otdela pozvonochnogo kanala. «Voprosy nejrohirurgii» im. N.N. Burdenko. 2012; 76 (3): 61-68 (in Russian).

36. T. Eneqvist et al., Lumbar surgery prior to total hip arthroplasty is associated with worse patientreported outcomes. Bone Joint J. 2017; 99-B: 759-65.

37. Vakulenko V. M., Klimovickij V. G., Bublik L. A. osobennosti diagnostiki i lechenija koksartroza na fone distroficheskih izmenenij v pojasnichno-krestcovom otdele pozvonochnika .Original'ni doslidzhennja. 2008; T. 9., 2: 1519 (in Russian).

38. Slinjakov L.Ju., Kavalerskij G.M., Makirov S.K., Chenskij A.D., Bobrov D.S. osobennosti ispol'zovanija intraoperacionnogo rentgenologicheskogo kontrolja pri maloinvazivnyh stabilizirujushhih operacijah na grudnom i pojasnichnom otdelah pozvonochnika.Vestnik Rossijskoj Voenno-medicinskoj akademii. SPb.: VMedA, 2009; 4 (28): 116-117 (in Russian).

39. Bresnahan, Lacey; Ogden, Alfred T.; Natarajan, Raghu N.; Fessler, Richard G. A biomechanical Evaluation of graded Posterior Element removal for treatment of lumbar stenosis: comparison of a Minimally invasive approach with two standard laminectomy techniques. Spine. 2009; Vol. 34., 1: 17-23.

40. Martin, C Ryan; Gruszczynski, Adam T.; Braunsfurth, Heike A.; Fallatah, Salah M.; O'Neil, Joseph; Wai, Eugene $\mathrm{K}$. The Surgical management of degenerative lumbar spondylolisthesis: A Systematic Review Spine. 2007; Vol.32, 16: 1791-1798.

41. Fu, Yi-Shan; Zeng, Bing-Fang; Xu, Jian-Guang long-term outcomes of two Different Decompressive techniques for lumbar Spinal Stenosis. Spine.2008; Vol.33, 5: 514-518.

42. Denisov A.O., Shil'nikov V.A., Barns S.A. Koksovertebral'nyj sindrom i ego znachenie pri jendoprotezirovanii tazobedrennogo sustava: (obzor lit.). Travmatologija i ortopedija Rossii. 2012; 1: 121-127 (in Russian).

43. Denisov A.O. Bolevoj sindrom posle jendoprotezirovanija tazobedrennogo sustava: avtoref. ... kand. med. nauk. SPb., 2010: 25. (in Russian).

44. Ahtjamov I.F., Guryleva M.Je., Juosef A.I. i dr. Ocenka vozmozhnyh parallelej mezhdu pokazateljami kachestva zhizni i kliniko-funkcional'nym sostojaniem $\mathrm{u}$ bol'nyh posle jendoprotezirovanija tazobedrennogo sustava. Vestnik travmatologii i ortopedii im. N.N. Priorova. 2008; 1: 75-79 (in Russian).

45. Vakulenko V.M. Differencirovannyj podhod k koksalgii na fone distroficheskih izmenenij v pojasnichno-krestcovom otdele pozvonochnika. Mezhdunarodnyj nevrologicheskij zhurnal. 2008; 2 (18): 152-156 (in Russian).

46. Ragozin A.O. Kliniko-biomehanicheskie aspekty optimizacii funkcii tazobedrennogo sustava pri jendoprotezirovanii: avtorof. dis. ... kand. med. nauk. M., 2004: 17. (in Russian).

47. Kacitadze T.A. Znachimost' biomehanicheskih pokazatelej pozvonochnika dlja prognoza ishoda lechenija displasticheskogo koksartroza. Georg. Med. News. 2004; 7-8: 13-15 (in Russian).

48. Kavalerskij G.M., Cherepanov V.G., Korkunov A.L., Lychagin A.V., Sereda A.P. Degenerativno-distroficheskie porazhenija pojasnichno-krestcovogo otdela pozvonochnika pri hip-spine sindrome: hirurgicheskoe lechenie Kafedra travmatologii i otropedii. 2013; 3 (7): 4-9 (in Russian).

49. Li, Gordon; Patil, Chirag G.; Lad, Shivanand P.; Ho, Chris; Tian, Wendy; Boakye, Maxwell. Effects of age and comorbidities on complication rates and adverse outcomes after lumbar laminectomy in elderly patients. Spine; 2008; Vol. 33, 11: 1250-1255. 